\title{
Grigore C. Moisil (1906 - 1973) and his School in Algebraic Logic
}

\author{
George Georgescu, Afrodita Iorgulescu, Sergiu Rudeanu
}

\begin{abstract}
We present in the paper a very concise but updated survey emphasizing the research done by Gr. C. Moisil and his school in algebraic logic.

Keywords: $n$-valued Lukasiewicz-Moisil algebra, $\theta$-valued Lukasiewicz-Moisil algebra, Post algebra
\end{abstract}

The mathematical logic is one of the domain in which the creative spirit of Gr.C. Moisil manifested plenary. His work in logic stands out by the the novelty, the variety and the depth of treated subjects. His first works are connected to the top results of the time and wear an algebraic seal. The young professor from Jassy came after a rich experience in mechanics and differential equations. Van der Waerden treatise of algebra has decisively influenced his entry in logic by the algebraic gate. In the same time, these works have a powerful philosophical imprint.

From this vast creation, the contributions in multiple-valued logics represent the part with the most intense impact on today researches.

The first system of multiple-valued logic was introduced by J. Łukasiewicz in 1920. Independently, E. Post introduced in 1921 a different multiple-valued logic. For Łukasiewicz, the motivation was of philosophical nature - he was looking for an interpretation of the concepts of possibility and necessity - while for Post, the research was intended as a natural mathematical generalization of bivalent logic.

In 1930, Łukasiewicz and Tarski studied a logic whose truth values are the real numbers from the interval $[0,1]$.

\section{Lukasiewicz-Moisil algebras}

In 1940, Gr. C. Moisil has defined the 3-valued and the 4-valued Lukasiewicz algebras and in 1942, the $n$ valued Łukasiewicz algebras $(n \geq 2)$. His goal was to algebrize Łukasiewicz's logic. Boolean algebras, algebraic models of classical logic, are particular cases of that new structures.

In the description of a logical system, the implication was traditionally the principal connector. The $n$-valent system of Łukasiewicz had as truth values the elements of the set

$$
L_{n}=\left\{0, \frac{1}{n-1}, \frac{2}{n-1}, \ldots, \frac{n-2}{n-1}, 1\right\}
$$

and was built around a new concept of implication, on which are based the definitions of the other connectors.

For Moisil, the basic structure is that of lattice, to which he adds a negation (getting the so called "De Morgan algebra") and also some unary operations (called by Moisil "chryssipian endomorphisms"), representing the "nuancing". The Łukasiewicz implication was let on a secondary plane and, in the case of an arbitrary valence, was completely lost.

Further axiomatizations were suggested by A. Monteiro, R. Cignoli, C. Sicoe, S. Rudeanu and others.

An example of A. Rose from 1956 established that for $n \geq 5$ the Łukasiewicz implication can no more be defined on a Łukasiewicz algebra. Consequently, only for $n=3$ and $n=4$ the structures introduced by Moisil are models for Łukasiewicz logic. The lost of implication has lead to another type of logic, called today "Moisil logic", distinct from Łukasiewicz system; the logic corresponding to $n$-valued Łukasiewicz-Moisil algebras was created by Moisil in 1964. The fundamental concept of Moisil logic is the nuancing.

Nowadays we feel it appropiate to call these algebras Lukasiewicz-Moisil algebras or LM algebras for short.

For complete information and references on Łukasiewicz-Moisil algebras see [25]

The work of Moisil on LM algebras covers two periods of time: a first period, during 1940-1942, when he introduces the n-valued LM algebras with negation and studies special classes of these structures, as centered and axed LM algebras and a second one, during 1954-1973, when he introduces the $\theta$-valued LM algebras without negation, applies multiple-valued logics to swiching theory and study algebraic properties of LM algebras (representation, ideals, reziduation). 
Moisil's works traced research directions for many Romanian and foreign mathematicians. In Argentina, at Bahia Blanca, Antonio Monteiro and his school (Roberto Cignoli, Luiz Monteiro, Luiza Iturrioz, Maurice Abad etc.) have contributed decisively to consolidate LM agebras as a domain of algebra of logic and to disseminate them in the mathematical world.

In his $\mathrm{PhD}$ thesis from 1969 [29], R. Cignoli makes a very deep study of $n$-valued Moisil algebras (the name he first gives to the $n$-valued Łukasiewicz algebras introduced by Moisil).

\section{1 n-valued Lukasiewicz-Moisil algebras}

The structure called "De Morgan algebra" was first studied by Moisil; the name was given by Antonio Monteiro [142]; a duplicate name is "quasi-Boolean algebra" given by A. Bialynicki-Birula and H. Rasiowa.

Definition 1.1. A De Morgan algebra is a structure

$$
(A, \vee, \wedge,-, 0,1)
$$

such that $(A, \vee, \wedge, 0,1)$ is a distributive lattice with 0 and 1 and the unary operation ${ }^{-}$, called negation, verifies:

(DMO) $\quad 1=0^{-}$,

(DM1) $\quad\left(x^{-}\right)^{-}=x$,

(DM2) $\quad(x \wedge y)^{-}=x^{-} \vee y^{-}$.

Remark 1.2. In a De Morgan algebra we also have:

(DM3) $\quad(x \vee y)^{-}=x^{-} \wedge y^{-}$.

Definition 1.3. Let $J=\{1,2, \ldots, n-1\}$.

An $n$-valued Lukasiewicz-Moisil algebra $(n \geq 2)$ or an $L M_{n}$ bf algebra for short is an algebra

$$
\mathcal{A}=\left(A, \vee, \wedge,-,\left(r_{j}\right)_{j \in J}, 0,1\right)
$$

of type $\left(2,2,1,(1)_{j \in J}, 0,0\right)$ such that:

(i) $\left(A, \vee, \wedge,{ }^{-}, 0,1\right)$ is a De Morgan algebra.

(ii) the unary operations $r_{1}, r_{2}, \ldots, r_{n-1}$ fulfil the following axioms: for every $x, y \in A$ and every $i, j \in J$,

(L1) $r_{j}(x \vee y)=r_{j} x \vee r_{j} y$,

(L2) $r_{j} x \vee\left(r_{j} x\right)^{-}=1$,

(L3) $r_{j} \circ r_{i}=r_{i}$,

(L4) $r_{j}\left(x^{-}\right)=\left(r_{n-j} x\right)^{-}$,

(L5) $r_{1} x \leq r_{2} x \leq \cdots \leq r_{n-1} x$

(L6) if $r_{j} x=r_{j} y$ for every $j \in J$, then $x=y$; this is the determination principle.

If $\mathcal{A}$ fulfils (i) and only (L1)-(L5) we shall say that $\mathcal{A}$ is an $L M_{n}$ pre-algebra.

Proposition 1.4. In every $L M_{n}$ algebra $\mathcal{A}$, the following properties are verified: for every $x, y \in A$ and every $j \in J$,

(L7) $r_{j}(x \wedge y)=r_{j} x \wedge r_{j} y$;

(L8) $r_{j} x \wedge\left(r_{j} x\right)^{-}=0$

(L9) $x \leq y$ if and only if $\left(r_{j} x \leq r_{j} y\right.$, for every $\left.j \in J\right)$;

(L10) $r_{1} x \leq x \leq r_{n-1} x$;

(L11) $r_{j} 0=0, r_{j} 1=1$;

(L12) Let $C(A)$ be the set of complemented elements of A, i.e.

$$
C(A)=\left\{x \in A \mid \exists x^{\prime} \in A, x \vee x^{\prime}=1, x \wedge x^{\prime}=0\right\} .
$$

Let $K_{j}$ be the set of all elements of $A$ left invariant by $r_{j}, j \in J$, i.e.

$$
K_{j}=\left\{x \in A \mid r_{j} x=x\right\}
$$


Then:

(i) $r_{j} x \in C(A)$, for every $j \in J, x \in A$ and

(ii) $C(A)=K_{j}$, for every $j \in J$;

(L12') $\left(C(A), \vee, \wedge,{ }^{-}, 0,1\right)$ is a Boolean algebra, where $x^{-}=x^{\prime}$;

(L12") If $z \in C(A)$, then for every $x \in A$ :

$x \wedge z=0 \Longleftrightarrow x \leq z^{-}$,

$z \vee x=1 \Longleftrightarrow z^{-} \leq x ;$

(L13) $x^{-} \vee r_{n-1} x=1$;

(L14) $x \wedge\left(r_{n-1} x\right)^{-}=0$.

Example 1.5. The algebra

$$
\mathcal{L}_{n}=\mathcal{L}_{n}^{\left(L M_{n}\right)}=\left(L_{n}, \vee, \wedge,-,\left(r_{j}\right)_{j \in J}, 0,1\right)
$$

where

$$
L_{n}=\left\{0, \frac{1}{n-1}, \frac{2}{n-1}, \ldots, \frac{n-2}{n-1}, 1\right\}
$$

and

$$
\left\{\begin{array}{l}
x \vee y=\max (x, y), \quad x \wedge y=\min (x, y), \quad x^{-}=1-x, \\
r_{j}\left(\frac{i}{n-1}\right)=\left\{\begin{array}{ll}
0, & \text { if } j+i<n, \\
1, & \text { if } j+i \geq n,
\end{array} \quad i \in\{0\} \cup J, j \in J,\right.
\end{array}\right.
$$

is an $L M_{n}$ algebra, that we shall call the canonical $L M_{n}$ algebra.

The proper subalgebras of $\mathcal{L}_{n}$ have the form:

$$
S=L_{n}-\bigcup_{x \in L_{n}-\{0\}}\left\{x, x^{-}\right\} .
$$

They are $L M_{n}$ algebras. (L12').

The smallest subalgebra of $\mathcal{L}_{n}$ (with respect to $\subseteq$ ) is $C\left(L_{n}\right)=\{0,1\}$, which is also a Boolean algebra, cf.

For instance, the subalgebras of

- $L_{3}$ are $L_{2}$ and $L_{3}$,

- $L_{4}$ are $L_{2}$ and $L_{4}$ and

- $L_{5}$ are $L_{2}, L_{3},\{0,1 / 4,3 / 4,1\}$ and $L_{5}$.

Remark 1.6. $\mathrm{LM}_{2}$ algebras coincide with Boolean algebras.

Proposition 1.7. In every $L M_{n}$ pre-algebra, the determination principle (L6) is equivalent to each of the following conditions: for every $x, y \in L$,

(a) $x \wedge\left(r_{j} x\right)^{-} \wedge r_{j+1} y \leq y$, for every $j \in J-\{n-1\}$;

(b) $x \wedge \bigwedge_{j=1}^{n-1}\left(\left(r_{j} x\right)^{-} \vee r_{j} y\right) \leq y$.

p (Representation theorem of Moisil)

Every $L M_{n}$ algebra can be embedded in a direct product of copies of the canonical $L M_{n}$ algebra $\mathcal{L}_{n}$.

Corollary 1.1. Every $L M_{n}$ algebra is a subdirect product of subalgebras of the canonical $L M_{n}$ algebra $\mathcal{L}_{n}$.

In 1968, Gr. C.Moisil introduced the $\theta$-valued Lukasiewicz algebras or $\mathbf{L} \mathbf{M}_{\theta}$ algebras for short (without negation), where $\theta$ is the order type of a chain with first and last element. The concept of $\theta$-valued Łukasiewicz algebra is obtained from that of $n$-valued, on the one hand, by dropping the negation ${ }^{-}$and on the other hand, by replacing the set $L_{n}$ by a totally ordered set $I$ with first and least elements and by adapting the axioms to this case; the Determination Principle is preserved. These structures were thought by Moisil as models of a logic with an infinity of nuances. According to a confession done by Moisil, he imagined $\mathrm{LM}_{\theta}$ algebras (without negation) long 
time ago, but the care of finding a strong motivation for them delayed the announcement; the motivation was found when Moisil met Zadeh's fuzzy set theory, in which he saw a confirmation of his old ideas.

In 1969, Marek and Traczyk [110] introduced the notion of generalized Lukasewicz algebra (with negation), in an attempt to generalize to the infinite case the $L M_{n}$ algebras; but their generalization is not a natural one.

In his $\mathrm{PhD}$ thesis from 1972 [64], G. Georgescu studied duality theory for Moisil's $L M_{\theta}$ algebras (without negation), the injective objects (and their characterization), monadic and poliadic algebras.

In his $\mathrm{PhD}$ thesis from 1981 [53], A. Filipoiu studied the $L M_{\theta}$ algebras (without negation) and their associated logic. He gives a representation theorem for $L M_{\theta}$ algebras by aids of $\theta$-valent Moisil field.

In his Master thesis from 1981 [12] also, L. Beznea studies a generalization of $L M_{\theta}$ algebras (without negation) obtained by eliminating the Determination Principle.

Later on, in his PhD theses from 1984 [21], V. Boicescu introduced and studied the $n$-valued LM algebras without negation, as a particular case of Moisil's $L M_{\theta}$ algebras (without negation).

Following the inverse way, A. Iorgulescu, in her PhD thesis from 1984 [90] also, introduced and studied a natural generalization of Moisil's $L M_{n}$ algebras to the infinite case, called $\theta$-valued LM algebras with negation or $L M_{\theta}$ algebras with negation for short; any $L M_{\theta}$ algebra with negation is a Moisil's $L M_{\theta}$ algebra without negation.

\section{Connection with logic}

Gr. C. Moisil invented LM algebras in order to create an algebraic structure playing the same role with respect to the multiple-valued logic as Boolean algebras play with respect to classical, bivalent logic. However, as shown by the example of A. Rose, this only happens for the cases $n=3$ and $n=4$.

The algebraic structures adequate to the infinite-valued logic of Łukasiewicz (truth valued in the real interval $[0,1])$ are the MV-algebras introduced by C.C. Chang in 1958 or, equivalently, the Wajsberg algebras introduced by Font, Rodriguez and Torrens in 1984; D. Mundici proved in in 1986 that MV algebras are categorically equivalent to lattice-ordered Abelian groups with strong unit.

R. Grigolia's $M V_{n}$ algebras, introduced in 1977, and Cignoli's proper Łukasiewicz algebras, introduced in 1982, are algebraic structures corresponding to $n$-valued logic of Łukasiewicz.

The logic corresponding to $L M_{n}$ algebras was created by Moisil himself in 1964. Eukasiewicz logic has implication as its primary connector, while Moisil logic is based on the idea of nuance, expressed algebraically by the Chrysippian endomorphisms. The "engine" of the latter logic is Moisil's Determination Principle, according to which an $n$-valued sentence is determined by its Boolean nuances. The Determination Principle realizes a transfer from the multiple-valued logic to the classical logic. This determination brings Moisil logic much closer to classical logic than Łukasiewicz logic. One could say that Moisil logic is derived from classical logic by the idea of nuancing. Algebraically, this tight relationship is expressed by the fundamental adjunction between the categories of Boolean and Łukasiewicz algebras.

V. Boicescu in 1971 and A. Filipoiu in 1981 introduced and studied logics appropiate to $L M \theta$ algebras without negation. (i.e. infinite-valued LM algebras).

A. Filipoiu generalized Smullyan's method of analytic tableaux to $\theta$-valued logic without negation and studied the $\theta$-valued predicate calculus as well, with applications to systems of recording and retrieval of information.

Łukasiewicz logic, Post logic and Moisil logic consitute the three directions in the classical theory of multiplevalued logic. Their corresponding algebraic models are $M V$ algebras, Post algebras and LM algebras.

\section{Connections with other structures of algebraic logic}

Moisil introduced in 1941 the centered $L M_{3}$ algebras.

Post algebras (cf. P. Rosenbloom (1942), G. Epstein (1960), T. Traczyk (1963)) turn out to be centered LM algebras, cf. R. Cignoli (1969) and G. Georgescu - C. Vraciu (1969). The $\theta$-valued Post algebras were studied by T. Traczyk (1967) and G. Georgescu (1971).

Gr. C. Moisil, R. Cignoli, L. Iturrioz, A. Monteiro and V. Boicescu studied LM algebras as particular cases of Heyting algebras. V. Boicescu also studied LM algebras as Stone algebras. 
$L M_{3}$ algebras and $L M_{4}$ algebras are polynomially equivalent to $M V_{3}$ algebras and $M V_{4}$ algebras, respectively, since they are the algebraic counterpart of the 3-valued Łukasiewicz logic and the 4-valued Łukasiewicz logic, respectively. D. Mundici was first to point out the equivalence between $L M_{3}$ algebras and $M V_{3}$ algebras, in 1989. Then A. Iorgulescu, in 1998-2000 [91] - [94], pointed out the isomorphism between the categories of $L M_{k}$ algebras and of $M V_{k}$ algebras, for $k=3,4$ and also studied the categories $\mathbf{L} \mathbf{M}_{n}$ and $\mathbf{M} \mathbf{V}_{n}$ for $n \geq 5$, showing that every $M V_{n}$ can be made into an $L M_{n}$ algebra. She then studied those $L M_{n}$ algebras that can be viewed as $M V_{n}$ algebras:

\subsection{Connections between $L M_{n}$ algebras and $M V_{n}$ algebras}

MV algebras were introduced by C.C. Chang, in 1958 [26]. A simplified list of axioms of MV algebras was given by Mangani [109], as follows:

Definition 3.1. An $M V$ algebra is an algebra

$$
\mathcal{A}=\left(A, \oplus,^{-}, 0\right)
$$

of type (2,1,0), where the following axioms are verified: for every $x, y, z \in A$,

(MV1) $(A, \oplus, 0)$ is an Abelian monoid,

(MV2) $\quad x \oplus 0^{-}=0^{-}$,

$(M V 3) \quad\left(x^{-}\right)^{-}=x$,

(MV4) $\quad\left(x^{-} \oplus y\right)^{-} \oplus y=\left(y^{-} \oplus x\right)^{-} \oplus x$,

where $x \cdot y=\left(x^{-} \oplus y^{-}\right)^{-}$.

Definition 3.2. For any $m \in \mathbb{N}$, we have:

(i) $0 x=0$ and $(m+1) x=m x \oplus x$,

(ii) $x^{0}=1$ and $x^{m+1}=x^{m} \cdot x$.

The $M V_{n}$ algebras were introduced by Revaz Grigolia in 1977 [87], as follows.

Definition 3.3. An $M V_{n}$ algebra $(n \geq 2)$ is an $M V$ algebra $\mathcal{A}=\left(A, \oplus,{ }^{-}, 0\right)$, whose operations fulfil the additional axioms:

(M1) $\quad(n-1) x \oplus x=(n-1) x$,

(M1') $\quad x^{n-1} \cdot x=x^{n-1}$

and, if $n \geq 4$, the axioms:

(M2) $\quad\left[(j x) \cdot\left(x^{-} \oplus[(j-1) x]^{-}\right)\right]^{n-1}=0$,

$\left(M 2^{\prime}\right) \quad(n-1)\left[x^{j} \oplus\left(x^{-} \cdot\left[x^{j-1}\right]^{-}\right)\right]=1$,

where $1<j<n-1$ and $j$ does not divide $n-1$.

Corollary 3.1. $M V_{2}$ algebras coincide with Boolean algebras.

Example 3.4. The $M V$ algebra $\mathcal{L}_{n}=\mathcal{L}_{n}^{\left(M V_{n}\right)}=\left(L_{n}, \oplus,{ }^{-}, 0\right)$, where

$$
L_{n}=\left\{0, \frac{1}{n-1}, \frac{2}{n-1}, \ldots, \frac{n-2}{n-1}, 1\right\}
$$

and for any $x, y \in L_{n}$ :

$$
x \oplus y=\min (1, x+y), \quad x \cdot y=\max (0, x+y-1), \quad x^{-}=1-x
$$

and

$$
x \vee y=\max (x, y), \quad x \wedge y=\min (x, y),
$$

is an $M V_{n}$ algebra. We shall call it the canonical $M V_{n}$ algebra.

Note that $B\left(L_{n}\right)=\{0,1\}$.

The subalgebras of $\mathcal{L}_{n}$ are of the form:

$$
S_{m}=\left\{0, \frac{K}{n-1}, \ldots, \frac{(m-2) K}{n-1}, 1\right\}
$$


where $K=\frac{n-1}{m-1}$, if $m-1$ divides $n-1$.

The subalgebras $S_{m}$ of $\mathcal{L}_{n}$ are isomorphic to $L_{m}=\left\{0, \frac{1}{m-1}, \ldots, \frac{m-2}{m-1}, 1\right\}$, if $m-1$ divides $n-1$, and they are $M V_{n}$ algebras.

Hence $\mathcal{L}_{m}=\left(L_{m}, \oplus, \cdot,{ }^{-}, 0,1\right)(m \leq n)$ is an $M V_{n}$ algebra if and only if $m-1$ divides $n-1$.

For instance, the subalgebras of:

- $L_{3}$ are $L_{2}$ and $L_{3}$,

- $L_{4}$ are $L_{2}$ and $L_{4}$ and

- $L_{5}$ are $L_{2}, L_{3}$ and $L_{5}$.

$\mathrm{p}$

Every $M V_{n}$ algebra is a subdirect product of subalgebras of the canonical $M V_{n}$ algebra $\mathcal{L}_{n}$.

D. Mundici was the first to prove in 1989 [152] that $M V_{3}$ algebras coincide with $L M_{3}$ algebras.

A. Iorgulescu has proved in 1998-2000 [91] - [94] that:

$1-M V_{4}$ algebras coincide with $L M_{4}$ algebras,

2 - the canonical $M V_{n}$ algebra coincides with the canonical $L M_{n}$ algebra $(n \geq 2)$,

3 - for $n \geq 5$, any $M V_{n}$ algebra is a $L M_{n}$ algebra,

4 - those $L M_{n}$ algebras which are $M V_{n}$ algebras, for every $n \geq 5$, are exactly Cignoli's proper $n$-valued Łukasiewicz algebras.

Here are for short the results 1-3:

To obtain the transformation of an $M V_{n}$ algebra into an $L M_{n}$ algebra, for any $n \geq 3$, Iorgulescu used Suchoń's transformation [174]:

Suchon defines Moisil operators $\left(\sigma_{j}\right)_{j \in J}\left(\sigma_{j}=r_{n-j}\right)$ of the canonical $L M_{n}$ algebra $(n \geq 3)$ starting from the Łukasiewiczian implication $\rightarrow$ and from the negation ${ }^{-}$. He puts

$$
B_{3}(x)=\left(x^{-}\right) \rightarrow x \text { and } B_{j+1}(x)=\left(x^{-}\right) \rightarrow B_{j}(x), j \geq 3 .
$$

Then he defines:

$$
\begin{aligned}
& \sigma_{1} x=B_{n}(x) \\
& \text { and for } 1<j \leq[n / 2], \quad \sigma_{j} x=\left\{\begin{array}{c}
\sigma_{n-1}\left(B_{l+1}(x)\right), l j \geq n-1 \\
\sigma_{l j}\left(B_{l+1}(x)\right), l j<n-1,
\end{array}\right.
\end{aligned}
$$

where $l=\max \{m \mid m(j-1)<n-1\}$,

$$
\text { while } \sigma_{n-j}(x)=\left(\sigma_{j}\left(x^{-}\right)\right)^{-}, \text {for } 1 \leq j \leq[n / 2] .
$$

Suchon's Moisil operators verify: $\quad \sigma_{1} \geq \sigma_{2} \geq \ldots \geq \sigma_{n-1}$.

Remark 3.5. If we want to use Suchon's construction, it is convenient to consider not the MV algebra $\left(A, \oplus,{ }^{-}, 0\right)$, but the Wajsberg algebra, $(A, \rightarrow,-, 1)$, introduced by J. M. Font, A. J. Rodriguez and A. Torrens in 1984; MV algebras and Wajsberg algebras are isomorphic structures:

- if $\mathcal{A}=(A, \rightarrow,-, 1)$ is a Wajsberg algebra and if we define

$$
\alpha(\mathcal{A})=\left(A, \oplus,^{-}, 0\right)
$$

by

$$
x \oplus y=x^{-} \rightarrow y, \quad 0=1^{-},
$$

then $\alpha(\mathcal{A})$ is an MV algebra.

- Conversely, if $\mathcal{A}=\left(A, \oplus,-{ }^{-}, 0\right)$ is an MV algebra and if we define

$$
\beta(\mathcal{A})=\left(A, \rightarrow,{ }^{-}, 1\right)
$$

by

$$
\begin{aligned}
x \rightarrow y & =x^{-} \oplus y, \\
1 & =0^{-},
\end{aligned}
$$

then $\beta(\mathcal{A})$ is a Wajsberg algebra.

- The maps $\alpha, \beta$ are mutually inverse. 
It follows immediately by (1) that

$$
B_{3}(x)=x \oplus x=2 x \quad \text { and } \quad B_{j+1}(x)=x \oplus B_{j}(x)=j x, j \geq 3 .
$$

By using Suchon's construction, Iorgulescu then gave the following

Definition 3.6. Let $\mathcal{A}=\left(A, \oplus,^{-}, 0\right)$ be an $M V_{n}$ algebra $(n \geq 3)$. Define

$$
\Phi^{S}(\mathcal{A})=\left(A, \vee, \wedge^{-},\left(r_{j}\right)_{j \in J}, 0,1\right)
$$

by

for $1<j \leq[n / 2], \quad l=\max \{m \mid m(j-1)<n-1\}$,

$$
\begin{gathered}
x \vee y=x \cdot y^{-} \oplus y, \quad x \wedge y=\left(x^{-} \vee y^{-}\right)^{-}, \\
r_{n-1} x=(n-1) x, \\
r_{n-j} x=\left\{\begin{array}{cc}
r_{1}(l x), & l j \geq n-1 \\
r_{n-l j}(l x), & l j<n-1,
\end{array}\right.
\end{gathered}
$$

$$
r_{j} x=\left(r_{n-j}\left(x^{-}\right)\right)^{-}, \quad 1 \leq j \leq[n / 2] .
$$

Proposition 3.7. If $\mathcal{L}_{n}$ is the canonical $M V_{n}$ algebra $(n \geq 3)$, then $\Phi^{S}\left(\mathcal{L}_{n}\right)$ is the canonical $L M_{n}$ algebra.

p If $\mathcal{A}$ is an $M V_{n}$ algebra $(n \geq 3)$, then $\Phi^{S}(\mathcal{A})$ is an $L M_{n}$ algebra.

Proposition 3.8. 1) Given the canonical $L M_{n}$ algebra $(n \geq 3)$

$$
\begin{gathered}
\mathcal{L}_{n}=\left(L_{n}, \vee, \wedge,{ }^{-},\left(r_{j}\right)_{j \in J}, 0,1\right), \\
\text { define } \Psi\left(\mathcal{L}_{n}\right)=\left(L_{n}, \oplus^{n},{ }^{-}, 0\right) \text { by : }
\end{gathered}
$$

if $n=2 k+1$,

$$
\begin{aligned}
x \oplus^{2 k+1} y= & \left(x \vee r_{2 k} y\right) \wedge\left(y \vee r_{2 k} x\right) \\
\wedge & \left(x^{*} \vee r_{2 k-1} y\right) \wedge\left(y^{*} \vee r_{2 k-1} x\right) \\
& \vdots \\
& \wedge\left(x^{(k-1) *} \vee r_{k+1} y\right) \wedge\left(y^{(k-1) *} \vee r_{k+1} x\right),
\end{aligned}
$$

if $n=2 k$,

$$
\begin{aligned}
x \oplus^{2 k} y= & \left(x \vee r_{2 k-1} y\right) \wedge\left(y \vee r_{2 k-1} x\right) \\
\wedge & \left(x^{*} \vee r_{2 k-2} y\right) \wedge\left(y^{*} \vee r_{2 k-2} x\right) \\
& \vdots \\
\wedge & \left(x^{(k-1) *} \vee r_{k} y\right) \wedge\left(y^{(k-1) *} \vee r_{k} x\right),
\end{aligned}
$$

where $x^{*}$ is the successor of $\mathrm{x}$ and

$$
x^{2 *}=\left(x^{*}\right)^{*} \quad, \quad x^{m *}=\left(x^{(m-1) *}\right)^{*}
$$

Then $\Psi\left(\mathcal{L}_{n}\right)$ is the canonical $M V_{n}$ algebra.

2) The maps $\Phi^{S}$, from Proposition 3.7, and $\Psi$ are mutually inverse.

Since for $n=3$, in the canonical $L M_{3}$ algebra $\mathcal{L}_{3}$, the operation $\oplus$ is:

$$
x \oplus y=\left(x \vee r_{2} y\right) \wedge\left(y \vee r_{2} x\right)
$$

and for $n=4$, in the canonical $L M_{4}$ algebra $\mathcal{L}_{4}$, the operation $\oplus$ is defined by:

$$
\begin{gathered}
x \oplus y=\left(x \vee r_{3} y\right) \wedge\left(y \vee r_{3} x\right) \wedge\left(x^{*} \vee r_{2} y\right) \wedge\left(y^{*} \vee r_{2} x\right)= \\
=\left(x \vee r_{3} y\right) \wedge\left(y \vee r_{3} x\right) \wedge\left(x^{-} \vee y^{-} \vee r_{2} x \vee r_{2} y\right),
\end{gathered}
$$

it follows that the transformation $\Psi$ is not polynomial for $n \geq 5)$.

Those $L M_{n}$ algebras which are $M V_{n}$ algebras (i.e. for which the transformation $\Psi$ is defined), for every $n \geq 5$, are exactly Cignoli's proper $n$-valued Łukasiewicz algebras [34], but the proof is very technical [94]. 


\section{Representation theorems}

Numerous representation theorems have been given for LM algebras. The first is due to Moisil himself and is reminiscent of the representation theorem for Boolean algebras: every $L M_{n}$ algebra can be embadded into a Cartesian power of $L_{n}$.

In a modern vision [25], every LM algebra is a subdirect product of subalgebras of the algebra $\operatorname{In}\left(I, L_{2}\right)$ of incresing functions from $I$ to $L_{2}$. In particular every $L M_{n}$ algebra is a subdirect product of subalgebras of $\mathcal{L}_{n}$ (Cignoli). $\mathcal{L}$ is a direct product of subalgebras of $\mathcal{L}_{n}$ if and only if it is complete and atomic (Boicescu 1984).

The representation by continuous functions studied by Cignoli, Boicescu and Filipoiu, means that for every $L M_{\theta}$ algebra without negation $\mathcal{L}$ there is a unique Boolean space $X$ such that $\mathcal{L}$ is isomorphic to the algebra of all continuous functions $f: X \rightarrow \operatorname{In}\left(I, L_{2}\right)$, where $\operatorname{In}\left(I, L_{2}\right)$ is endowed with the topology having as basis the principal ideals and the principal filters generated by the characteristic functions of the sets $\{k \mid k>\alpha\}, \alpha \in \theta$.

The representation of $L M_{\theta}$ algebras without or with negation by Moisil fields of sets is due to Filipoiu. The Stone duality was extended from Boolean algebras to $L M_{\theta}$ algebras without or with negation with the aid of a suitable concept called $L M_{\theta}$-valued Stone space (Cignoli, Georgescu, Iorgulescu), while the Priestley duality is based on a suitable adaptation of the concept of Priestley space (Filipoiu).

The representation of LM algebras as algebras of fuzzy sets was studied by D. Ponasse, J.L. Coulon and J. Coulon, S. Ribeyre and S. Rudeanu.

The representation of $L M_{n}$ algebras by $L M_{3}$ algebras is legitimated by the "good" properties of the latter and was studied by A. Monteiro, L. Monteiro, F. Coppola, V. Boicescu and A. Iorgulescu.

\section{Categorial aspects}

The Stone and Priestley dualities are in fact equivalences of categories. Other categorial properties of LM algebras were studied. Here are a few samples.

The association of $L$ with the Boolean alegebra $C(L)$ of complemented elements of $L$ is extended to a functor $C: \mathbf{L M} \theta \rightarrow \mathbf{B}$, while the association of a Boolean algebra $B$ with the algebra $\operatorname{In}(I, B)$ is extended to a functor $T$ : $\mathbf{B} \rightarrow \mathbf{L M} \theta$. Then $C$ and $T$ are adjoint functors, $C$ is faithful and $T$ is fully faithful. This yields in particular the representation theorem of Moisil. The construction of the functors $C$ and $T$ was given by Moisil himself.

The injective and projective objects have also been studied, for instance, an $L M_{\theta}$ algebra is injective if and only if it is a complete Post algebra (whose center is a complete Boolean algebra), cf. L. Monteiro, R. Cignoli, G. Georgescu and C. Vraciu, V. Boicescu.

\section{Ideals and congruences}

The study of the appropiate ideal and congruence theory for LM algebras was undertaken by Gr. C. Moisil, A. Monteiro, R. Cignoli, C. Sicoe. V. Boicescu introduced the concepts of $\theta$-ideal and $\theta$-congruence, the prime spectre. For instance, in the case of $L M_{n}$ algebras without negation, the congruence lattice of $\mathcal{L}$ is a Boolean algebra (a Stone algebra) if and only if $\mathcal{L}$ is finite $(C(L)$ is a complete Boolean algebra).

\section{Monadic and polyadic algebras}

L. Monteiro and G. Georgescu studied the generalization to LM algebras of the monadic and polyadic Boolean algebras introduced by P.R. Halmos. Sample results: the representation of monadic LM algebras by functional monadic LM algebras and the semantic completeness for polyadic LM algebras. A paper of G. Geogescu, A. Iorgulescu and I. Leuştean investigates monadic $M V_{n}$ algebras and closed $M V_{n}$ algebras. 


\section{Miscellanea}

Various other topics have also been studied. Thus:

V. Boicescu proved that the lattice of equational subclass of $L M_{n}$ is a finite Heyting algebra. The study of atomic algebras and the characterization of simple algebras as subalgebras of $\operatorname{In}\left(I, L_{2}\right)$ and the property that $L M_{n}$ algebras without negation form an equational class, are also due to Boicescu. The study of irredundant algebras and of exactly $n$-valued algebras is due to Boicescu as well.

A. Iorgulescu introduced and studied $m$-complete $L M_{\theta}$ algebras with negations, generalizing many of the properties of $m$-complete Boolean algebras.

G. Georgescu and I. Leuştean studied probabilities on LM algebras.

L. Beznea studied a generalization of LM algebras, obtained by dropping the determination principle.

Let us also mention M. Sularia's theory of D algebras. These structures are subdirect products between a Heyting algebra and a Brouwer algebra and represent the algebraic counterpart of a logic of problem solving.

In [44], [48], the authors study Łukasiewicz BCK algebras endowed with Moisil oparators.

In [163], [164], C. Sanza introduced and studied (monadic) $n \times m$-valued Łukasiewicz algebras with negation.

In a very recent paper [103], I. Leuştean proposes a unifying framework for $L M_{n}$ algebras, MV algebras and Post algebras; essentially, an $L M_{n+1}$ algebra is charcterized by a string of $n$ Boolean ideals of his Boolean center. The necessary and sufficient conditions are given that such a string must satisfy to define a $M V_{n+1}$ algebra or a Post algebra of order $n+1$. This result could be seen as a generalization of Moisil's Determination Principle. As an application, in paper [75], some special Cauchy completions of $M V_{n+1}$ algebras are characterized by using the properties of corresponding strings of Boolean ideals.

In another very recent paper [75], G.Georgescu and A. Popescu introduced the notion of $n$-nuanced MV algebra, by performing a Łukasiewicz-Moisil nuancing construction on top of MV-algebras. These structures extend both MV-algebras and Łukasiewicz-Moisil algebras, thus unifying two important types of structures in the algebra of logic. On a logical level, $n$-nuanced MV algebras amalgamate two distinct approaches to manyvaluedness: that of the infinitely valued Łukasiewicz logic, more related in spirit to the fuzzy approach, and that of Moisil $n$-nuanced logic, which is more concerned with nuances of truth rather than truth degrees. They study $n$-nuanced MV algebras mainly from the algebraic and categorial points of view and also consider some basic model-theoretic aspects. The relationship with a suitable notion of $n$-nuanced ordered group via an extension of the $\Gamma$ construction is also analyzed:

\section{1 n-nuanced MV algebras}

Usually, MV algebras are defined only in terms of $\oplus,{ }^{-}$and 0 . However, in order to point out the symmetry of these structures, the authors prefered the following slightly redundant definition:

Definition 8.1. An MV algebra is a structure $\left(A, \oplus, \odot,{ }^{-}, 0,1\right)$, satisfying the following axioms:

(MV1') $\quad(A, \oplus, 0)$ and $(A, \odot, 1)$ are commutative monoids,

(MV2') $\quad x \odot 0=0$ and $x \oplus 1=1$,

$\left(M V 3^{\prime}\right) \quad\left(x^{-}\right)^{-}=x$,

$\left(M V 4^{\prime}\right) \quad(x \oplus y)^{-}=x^{-} \odot y^{-}$,

$\left(M V 5^{\prime}\right) \quad\left(x \odot y^{-}\right) \oplus y=\left(y \odot x^{-}\right) \oplus x$.

Definition 8.2. A generalized De Morgan algebra is a structure $\mathcal{L}=\left(L, \oplus, \odot,{ }^{-}, 0,1\right)$, where $\oplus$, $\odot$ are binary operations, ${ }^{-}$is a unary operation, and 0,1 are constants such that the following conditions hold:

(i) $(L, \oplus, 0),(L, \odot, 1)$ are commutative monoids;

(ii) $\quad(x \oplus y)^{-}=x^{-} \odot y^{-}$and $\left(x^{-}\right)^{-}=x$ for all $x, y \in L$; .

Remark 8.3. If $\mathcal{L}$ is a generalized De Morgan algebra, then $(x \odot y)^{-}=x^{-} \oplus y^{-}$for all $x, y \in L$.

Definition 8.4. An n-nuanced MV-algebra $\left(N M V_{n}\right.$ algebra for short) is a structure

$$
\mathcal{L}=\left(L, \oplus, \odot,^{-}, r_{1}, \ldots, r_{n-1}, 0,1\right)
$$

such that $(L, \oplus, \odot,-, 0,1)$ is a generalized De Morgan algebra and $r_{1}, \ldots, r_{n-1}$ satisfy the following axioms:

(A0) $r_{i} x \oplus\left(\left(r_{i} x\right)^{-} \odot r_{i} y\right)=r_{i} y \oplus\left(\left(r_{i} y\right)^{-} \odot r_{i} x\right)$, for $i \in\{1, \ldots, n-1\}$, 
(A1) $r_{i}(x \oplus y)=r_{i} x \oplus r_{i} y, \quad r_{i}(x \odot y)=r_{i} x \odot r_{i} y, \quad r_{i}(0)=0, r_{i}(1)=1$, for $i \in\{1, \ldots, n-1\}$,

(A2) $r_{i} x \oplus\left(r_{i} x\right)^{-}=1, r_{i} x \odot\left(r_{i} x\right)^{-}=0$, for $i \in\{1, \ldots, n-1\}$,

(A3) $r_{i} \circ r_{j}=r_{j}$, for $i, j \in\{1, \ldots, n-1\}$,

(A4) $r_{i}\left(x^{-}\right)=\left(r_{n-i} x\right)^{-}$, for $i \in\{1, \ldots, n-1\}$,

(A5) (Determination Principle:) if $r_{i} x=r_{i} y$ for each $i \in\{1, \ldots, n-1\}$, then $x=y$,

(A6) $r_{1} x \leq r_{2} x \leq \ldots \leq r_{n-1} x$.

Remark 8.5. $N M V_{n}$ algebras provide a common generalization of $M V$ - and Łukasiwicz-Moisil algebras. Indeed, - if $n=2$, then, because of the Determination Principle, $r_{1}$ is the identity, thus an $N M V_{n}$ algebra can be identified with an $M V$-algebra;

- if $\left(L, \oplus, \odot,{ }^{-}, 0,1\right)$ is a De Morgan algebra, then $\left(L, \oplus, \odot,{ }^{-}, r_{1}, \ldots, r_{n-1}, 0,1\right)$ becomes an LM $M_{n}$ algebra.

Example 8.6. Let $\mathcal{A}=\left(A, \oplus, \odot,^{-}, 0,1\right)$ be an $M V$-algebra. Consider the set

$$
T(A)=\left\{\left(x_{1}, \ldots, x_{n-1}\right) \in A^{n-1} \mid x_{1} \leq \ldots \leq x_{n-1}\right\} .
$$

Since $A^{n-1}$ is an $M V$-algebra (with operations taken component-wise from $A$ ) and $T(A)$ is closed under the operations $\mathbf{0}, \mathbf{1}, \oplus, \odot$ (where $\mathbf{0}$ and $\mathbf{1}$ are the constant vectors), then we can consider these operations on $T(A)$. We furthermore define ${ }^{-}, r_{1}, \ldots, r_{n-1}$ by:

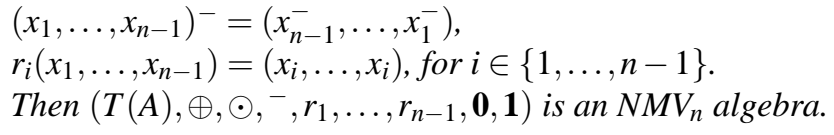

Define

$$
M(L)=\left\{x \in L \mid r_{i} x=x \text { for all } i \in\{1, \ldots, n-1\}\right\} .
$$

Then $M(L)$, together with the operations $\oplus, \odot,^{-}, 0,1$ induced by $\mathcal{L}$, is an MV algebra, called the MV-center of $\mathcal{L}$.

In the MV-algebra $M(L)$ we have a canonical order $\leq$. Let us define an extension of this order to $L$ by:

$x \leq y$ iff, for each $i \in\{1, \ldots, n-1\}, r_{i} x \leq r_{i} y$.

Because of the Determination Principle, this is indeed an order and because of (A3), it is indeed an extension of the order on $M(L)$. Moreover, the compatibility properties listed in the following lemma are obvious:

Proposition 8.7. The following properties are true in a $\mathcal{L}$ :

(1) 0 is the greatest and 1 is the least element in L w.r.t. $\leq$;

(2) for each $x, y \in L, x \leq y$ iff $y^{-} \leq x^{-}$;

(3) for each $x, x^{\prime}, y, y^{\prime} \in L$, if $x \leq x^{\prime}$ and $y \leq y^{\prime}$, then $x \oplus y \leq x^{\prime} \oplus y^{\prime}$ and $x \odot y \leq x^{\prime} \odot y^{\prime}$,

(4) $r_{1} x \leq x \leq r_{n-1} x$, for any $x \in L$,

(5) for $x, y \in L$, if $x \oplus y=1$ and $x \odot y=0$, then $x, y \in M(L)$ and $y=x^{-}$,

(6) $M(L)=\left\{x \in L \mid x \oplus x^{-}=1^{\prime}, \quad x \odot x^{-}=0\right\}$.

\section{Applications to switching theory}

Whereas Boolean algebra is a suitable tool for the study of networks made up of binary devices, the study of networks involving multi-positional devices and the so-called hazard and race phenomena have imposed the use of other algebraic tools, namely Galois fields, Łukasiewicz-Moisil algebras and the theory of discrete functions.

Moisil investigated circuits involving devices such as polarized relays with unstable neutral, ordinary relays under low self-maintaining current, valves, resistances, multi-positional relays, as well as transistors and other electronic devices. See also [125]. Moisil has described the operation of such devices by characteristic equations of the form $x_{k+1}=\varphi\left(\xi_{k}, x_{k}\right)$, where the variable $x$ associated with the relay contact takes values in $L_{n}$, where $n \leq 5$ 
depends on the type of the relay, $\xi \in L_{2}$ is a variable associated with the current and the index $k$ or $k+1$ indicates the value of the corresponing variable at time $t=k$ or $t=k+1$, respectively.

The synthesis problem consists in designing a circuit made up of several relays and whose operation be described by a given equation of the form

$$
X_{k+1}=F\left(A_{k}, X_{k}\right),
$$

where $X$ is the vector of the variables $x$ associated with the relays of the circuit, $A$ is the input vector and the meaning of the index $k$ or $k+1$ is the same as above. To solve this problem, Moisil notices the crucial point that the structure of such a circuit is determined by the expression of a function $G$ which satisfies the identity

$$
\Xi=G(A, X)
$$

where $\Xi$ is the vector of the variables $\xi$ associated with the relay of the circuit. So if

$$
X_{k+1}=\operatorname{Phi}\left(\Xi_{k}, X_{k}\right)
$$

is the vector form of the characteristic equations of the relays of the circuit, it follows from (14) and (15) that

$$
F\left(A_{k}, X_{k}\right)=\Phi\left(\Xi_{k}, X_{k}\right)
$$

for any $k$. Therefore (15) transforms (17) into the identity

$$
F(A, X)=\Phi(G(A, X), X)
$$

and Moisil's method for solving the synthesis problem consists in solving the functional equation (18) with respect to $G$.

\section{References}

[1] A. Amroune, Representation des algèbres de Łukasiewicz $\theta$-valentes involutives par des structures floues, BUSEFAL (Institut de Recherche en Informatique de Toulouse), 43, 1990, 5-11.

[2] M. Abad, Three valued Łukasiewicz algebras with an additional operation, Rev. Union Mat. Argentina, 32 , $1985,107-117$.

[3] M. Abad, A. Figallo, Characterization of three-valued Łukasiewicz algebras, Rep. Math. Logic, 18, 1984, 47-59.

[4] M. Abad, L. Monteiro, On three-valued Moisil algebras, Logique et Analyse, 27, 1984, 407-414.

[5] I. Bădele, V. Boicescu, Sur les extensions des algèbres de Łukasiewicz, C.R. Acad. Sci. Paris, 269, 1969, 313-315.

[6] D. Becchio, Axiomatisation d'une logique trivalente Łukasiewiczienne, 1971, Manuscript.

[7] D. Becchio, Nouvelle démonstration de la complétude du système de Wajsberg axiomatisant la logique trivalente de Łukasiewicz, C.R. Acad. Sci. Paris, 275, 1972, 679-681.

[8] D. Becchio, Sur les définitions des algèbres trivalentes de Łukasiewicz donées par A. Monteiro, Logique et Analyse, 63-64, 1973, 339-344.

[9] D. Becchio, Algèbres de Heyting, algèbres de Brouwer et algèbres trivalentes de Łukasiewicz, Logique et Analyse, 21, 1978, 237-248.

[10] D. Becchio, Logique trivalente de Łukasiewicz, Ann. Sci., Univ. Clermont-Ferrand, 16, 1978, 38-89.

[11] D. Becchio, L. Iturrioz, Sur une définition des algèbres de Łukasiewicz et de Post d'ordre $n$, Demonstratio Math., 11, 1978, 1083-1094.

[12] L. Beznea, $\theta$-valued Moisil algebras and dual categories (Romanian), Master Thesis, University of Bucarest, 1981. 
[13] V. Boicescu, Sur la représentation des algèbres de Łukasiewicz $\theta$-valentes, C.R. Acad. Sci. Paris, 270, 1970, 4-7.

[14] V. Boicescu, Sur les algèbres de Łukasiewicz, Logique,Automatique, Informatique, Editions de l'Academie de la R.S.Roumanie, 1971, 71-89.

[15] V. Boicescu, Sur les systèmes déductifs dans la logique $\theta$-valente, Publ. Dép. Math. Lyon, 8, 1971, 123-133.

[16] V. Boicescu, On Łukasiewicz algebras (Romanian), In: Probleme de logică, vol.IV, Ed. Academiei R.S.Romania, Bucharest, 1972, 245-276.

[17] V. Boicescu, On $\theta$-valued logics (Romanian), In: Probleme de Logică, vol. V, 1973, Edit. Acad. R.S.Romania, 241-255.

[18] V. Boicescu, Sur une logique polivalente, Rev. Roum. Sci. Soc., sér. Philos. et Logique, 17, 1973, 393-405.

[19] V. Boicescu, Researches in Łukasiewicz algebras, Rev. Roum. Sci. Soc., sér. Philos. et Logique, 20, 1976, 197-200.

[20] V. Boicescu, Extensions of homomorphisms of Lulasiewicz algebras with bounding semimorphisms, Rev. Roum. Sci. Soc., sér. Philos et Logique, 23, 1979, 367-370.

[21] V. Boicescu, Contributions to the study of Łukasiewicz algebras (Romanian), Ph.D. Thesis, University of Bucharest, 1984.

[22] V. Boicescu, Irredundant $n$-valued Moisil algebras, Discrete Math., 71, 1988, 197-204.

[23] V. Boicescu, G. Georgescu, Les algèbres de Łukasiewicz centrées et axées, Rev. Roum. Math. Pures et Appl., 15, 1970, 675-681.

[24] V. Boicescu, A. Iorgulescu, Current research in the field of Łukasiewicz-Moisil algebras (Romanian), Studii Cerc. Mat., 39, 1987, 97-106.

[25] V. Boicescu, A. Filipoiu, G. Georgescu, S. Rudeanu, Łukasiewicz-Moisil algebras, Annals of Discrete Mathematics, 49, 1991, North-Holland.

[26] C.C. Chang, Algebraic analysis of many valued logics, Trans. Amer. Math. Soc., 1958, 88, 467-490.

[27] R. Cignoli, Boolean elements in Łukasiewicz algebras. I. Proc. Japan Acad., 41, 1965, 670-675.

[28] R. Cignoli, Un teorema de representation para algebras de Łukasiewicz trivalentes, Rev. Union Mat. Argentina, 23, 1966, 41.

[29] R. Cignoli, Algebras de Moisil de orden $n$, Ph.D. Thesis, Universidad Nacional del Sur, Bahia Blanca, 1969.

[30] R. Cignoli, Moisil algebras, Notas de Logica Matematica, Inst. Mat., Univ. National del Sur, Bahia-Blanca, No. 27, 1970.

[31] R. Cignoli, Representation of Łukasiewicz algebras and Post algebras by continuous functions, Colloq. Math. 24, 1972, 127-138.

[32] R. Cignoli, Topological representation of Łukasiewicz and Post algebras, Notas de Logica Matematica, Inst. Mat. Univ. National del Sur, Bahia-Blanca, No. 33, 1974.

[33] R. Cignoli, Coproducts in the categories of Kleene and three-valued Łukasiewicz algebras, Studia Logica, 39, 1979, 237-245.

[34] R. Cignoli, Proper $n$-Valued Łukasiewicz Algebras as S-Algebras of Łukasiewicz n-Valued Propositional Calculi, Studia Logica, 41, 1982, 3-16.

[35] R. Cignoli, An algebraic approach to elementary theories based on $n$-valued Łukasiewicz logics, Z. Math. Logik u. Grundl. Math., 30, 1984, 87-96. 
[36] R. Cignoli, M.S. De Gallego, The lattice structure of 4-valued Łukasiewicz algebras, J. Symbolic Logic, 46, No. 1, 1981, 185.

[37] R. Cignoli, M.S. De Gallego, The lattice structure of some Łukasiewicz algebras, Algebra Universalis, 13, 1981, 315-328.

[38] R. Cignoli, A. Monteiro, Boolean elements in Łukasiewicz algebras. II., Proc. Japan Acad., 41, 1965, 676680.

[39] R. Cignoli and D. Mundici, An elementary proof of Chang's completeness theorem for the infinite-valued calculus of Łukasiewicz, Studia Logica, to appear.

[40] R. Cignoli, I.M.L. D’Ottaviano, D. Mundici, Algebraic Foundations of many-valued Reasoning, Kluwer 2000, Volume 7.

[41] J. Coulon, J.L. Coulon, A propos de la représentation des algèbres de Łukasiewicz et des algèbres booléiennes floues, Rev. Roum. Math. Pures et Appl., 34, 1989, 403-411.

[42] J. Coulon, J.L. Coulon, Un nouveau resultat concernant la representation d'une algèbre de Łukasiewicz involutive dans l'algèbre des parties floues d'une structure floue involutive, Rev. Roumaine Math. Pures et Appl., 38, 1993, 319-326.

[43] A. Figallo, J. Tolosa, Algebras de Łukasiewicz trivalente, Univ. Nac. de San Juan, 1982.

[44] A. Figallo Jr., M. Figallo and A. Ziliani, Free $(n+1)$-valued Łukasiewicz BCK algebras, Demonstratio Mathematica, XXXVII, 2, 2004, 245-254.

[45] A.V. Figallo, I. Pascual, A. Ziliani, Notes on monadic $n$-valued Łukasiewicz algebras, Math. Bohem., 3, 129, 2004, 255-271.

[46] A.V. Figallo, C. Sanza , A. Ziliani, Functional monadic $n$-valued Łukasiewicz algebras, accepted by Mathematica Bohemica.

[47] A.V. Figallo, I. Pascual, A. Ziliani, Subdirectly irreducible monadic Łukasiewicz-Moisil algebras, Manuscript.

[48] A.V. Figallo, Łukasiewicz BCK-algebras endowed with Moisil operators, Manuscript.

[49] A.V. Figallo, A. Figallo Jr., M. Figallo, A. Ziliani, Łukasiewicz residuation algebras with infimum, Manuscript.

[50] A. Filipoiu, Analytic tableaux for $\theta$-valued propositional logic, Math. Seminar Notes, 6, 1978, 517-526.

[51] A. Filipoiu, Representation theorems for Łukasiewicz algebras, Discrete Math., 27, 1979, 107-110.

[52] A. Filipoiu, Representation of Łukasiewicz algebras by means of ordered Stone spaces, Discrete Math., 30 , 1980, 111-116.

[53] A. Filipoiu, $\theta$-valued Łukasiewicz-Moisil algebras and logics (Romanian), Ph.D. Thesis, Univ. of Bucharest, 1981.

[54] A. Filipoiu, Representation theorems for $\theta$-valued Łukasiewicz algebras, Discrete Math., 33, 1981, 21-27.

[55] A. Filipoiu, Some remarks on the representation theorem of Moisil, Discrete Math., 33, 1981, 163-170.

[56] J. M. Font, A. J. Rodriguez, A. Torrens, Wajsberg Algebras, Stochastica, VIII, 1, 5-31, 1984.

[57] G. Georgescu, Algébres de Łukasiewicz complètes, C.R. Acad. Sci. Paris, 269, 1969, 1181-1184.

[58] G. Georgescu, The centered epimorphisms and the construction of the tensor product in Łuk, Rev. Roum. Math. Pures Appl., 15, 1970, 693-709.

[59] G. Georgescu, n-valued complete Łukasiewicz algebras, Rev. Roum. Math. Pures Appl., 16, 1971, 41-50. 
[60] G. Georgescu, The $\theta$-valued Łukasiewicz algebras. I., Rev. Roum. Math. Pures Appl., 16, 1971, $195-209$.

[61] G. Georgescu, Algebras de Łukasiewicz de orden $\theta$. II., Rev. Roum. Math. Pures Appl., 16, 1971, 363-369.

[62] G. Georgescu, Les algèbres de Łukasiewicz $\theta$-valentes, In: Logique, Automatique, Informatique, Edit. Acad. R.S.Romania, Bucharest, 1971, 99-169.

[63] G. Georgescu, The $\theta$-valued Łukasiewicz algebras. III. Duality theory., Rev. Roum. Math. Pures Appl., 16, $1971,1365-1390$.

[64] G. Georgescu, Algebre Łukasiewicz $\theta$-valente, Ph.D. Thesis, Math. Institute, Bucharest, mai 1972.

[65] G. Georgescu, Représentation des algèbres de Łukasiewicz $\theta$-valentes polyadiques, C. R. Acad. Sci. Paris, A-B, 274, 1972, A944-A946.

[66] G. Georgescu, Reprezentarea algebrelor Łukasiewicz poliadice local finite de grad infinit, Studii Cerc. Mat., 24, 1972, 1107-1117.

[67] G. Georgescu, Some remarks on the polyadic Łukasiewicz algebras, Rev. Roum. Mat. Pures Appl., 22, 1977, 641-648.

[68] G. Georgescu, On the homogeneous-universal Łukasiewicz algebras, Rev. Roum. Mat. Pures Appl., 23, 1978, 29-32.

[69] G. Georgescu, A. Iorgulescu, Pseudo-MV Algebras: a Noncommutative Extension of MV Algebras, The Proceedings of the Fourth International Symposium on Economic Informatics, Bucharest, Romania, May 1999, 961-968.

[70] G. Georgescu, I. Leuştean, Towards a probability theory based on Moisil logic, Soft Computing, 2000, 4, No.1, 19-26.

[71] G. Georgescu, A. Iorgulescu, Pseudo-BL algebras: A noncommutative extension of BL algebras, Abstracts of The Fifth International Conference FSTA 2000, Slovakia, February 2000, 90-92.

[72] G. Georgescu, A. Iorgulescu, Pseudo-BCK algebras: An extension of BCK algebras, Proceedings of DMTCS'01: Combinatorics, Computability and Logic, Springer, London, 2001, 97-114.

[73] G. Georgescu, I. Leuştean, Probabilities on Łukasiewicz-Moisil algebras, International Journal of Approximate Reasoning, 1998, 18, No.3-4, 201-215.

[74] G. Georgescu, I. Leuştean, Conditional probabilities on Łukasiewicz-Moisil algebras, Analele Universitatii Bucuresti, 1998, 47, 55-64.

[75] G. Georgescu., I. Leuştean, A. Popescu, Order convergence and distance on Łukasiewicz-Moisil algebras, Multiple Valued Logic, to appear.

[76] G. Georgescu, A. Popescu, A common generalization for algebras and Łukasiewicz-Moisil algebras, submitted.

[77] G. Georgescu, C. Vraciu, Le spectre maximal dŠune algèbre de Łukasiewicz, C.R. Acad. Sci. Paris, 268, 1969, 928-929.

[78] G. Georgescu, C. Vraciu, n-valued centered Łukasiewicz algebras, Rev. Roum. Math. Pures Appl., 14, 1969, 712-723.

[79] G. Georgescu, C. Vraciu, Sur les algèbres de Łukasiewicz centrées, C.R. Acad. Sci. Paris, 268, 1969, 9981000 .

[80] G. Georgescu, C. Vraciu, Sur le spectre maximal d'une algèbre de Łukasiewicz, Publ. Dép. Math. Lyon, 6, $1969,42-54$.

[81] G. Georgescu, C. Vraciu, Sur les épimorphismes centrées des algèbres de Łukasiewicz, C.R. Acad. Sci. Paris, 269, 1969, 4-6. 
[82] G. Georgescu, C. Vraciu, On the characterization of centered Łukasiewicz algebras, J. Algebra, 16, 1970, 486-495.

[83] G. Georgescu, C. Vraciu, Monadic Boolean algebras and monadic Łukasiewicz algebras (Romanian), Studii Cerc. Mat., 23, 1971, 1025-1048.

[84] G. Georgescu, C. Vraciu, La dualité des algèbres de Post $\theta$-valentes, J. Algebra, 21, 1972, 74-86.

[85] H. Goldberg, H. Leblanc, G. Weaver, A strong completeness theorem for 3-valued logic, Notre Dame J. Formal Logic, 15,1974, 325-332.

[86] M. Greniewski, Using three valued logics in the theory of swiching theory (Romanian). I. Realizarea cu circuite a funcţiilor fundamentale, Comunic. Acad. R.P.R., 6, 1956, 225-229.

[87] R. Grigolia, Algebraic analysis of Łukasiewicz-Tarski’s $n$-valued logical systems, in: Selected Papers on Łukasiewicz Sentential Calculi (R. Wójcicki and G. Malinowski, Eds.), Polish Acad. Of Sciences, Ossolineum, Wroclaw, 1977, 81-92.

[88] A. Iorgulescu, On the construction of three-valued Łukasiewicz-Moisil algebras, Discrete Math., 47, 1984, 213-227.

[89] A. Iorgulescu, Functors between categories of three-valued Łukasiewicz-Moisil algebras, Discrete Math., 49, 1984, 121-131.

[90] A. Iorgulescu, $(1+\theta$ )-valued Łukasiewicz-Moisil algebras with negation (Romanian), Ph.D. Thesis, Univ. of Bucharest, 1984.

[91] A. Iorgulescu, Connections between $M V_{n}$ algebras and $n$-valued Łukasiewicz-Moisil algebras - I, Discrete Mathematics, 181 (1-3), 1998, 155-177.

[92] A. Iorgulescu, Connections between $M V_{n}$ algebras and $n$-valued Łukasiewicz-Moisil algebras - II, Discrete Mathematics, 202, 1999, 113-134.

[93] A. Iorgulescu, Connections between $M V_{n}$ algebras and $n$-valued Łukasiewicz-Moisil algebras -III, Manuscript.

[94] A. Iorgulescu, Connections between $M V_{n}$ algebras and $n$-valued Łukasiewicz-Moisil algebras - IV, Journal of Universal Computer Science, vol. 6, no I , 2000, 139-154.

[95] L. Iturrioz, Axiomas para el calculo proposicional trivalente de Łukasiewicz, Rev. Union Mat. Argentina, $22,1965,150$.

[96] L. Iturrioz, Sur une classe particulière d'algèbres de Moisil, C. R. Acad. Sci. Paris, 267, 1968, 585-588.

[97] L. Iturrioz, Les algèbres de Heyting-Brouwer et de Łukasiewicz trivalentes, Notre Dame J. Formal Logic, $17,1976,119-126$.

[98] L. Iturrioz, Algèbres de Łukasiewicz symétriques, Publ. Dép. Math. Lyon, 13, 1976, 73-96.

[99] L. Iturrioz, Łukasiewicz and symmetrical Heyting algebras, Z. Math. Logik u. Grund.Math., 23, 1977, 131136.

[100] L. Iturrioz, Two characteristic properties of monadic three-valued Łukasiewicz algebras, Rep. Math. Logic, $8,1977,63-68$.

[101] L. Iturrioz, An axiom system for three-valued Łukasiewicz propositional calculus, Notre Dame J. Formal Logic, 18, 1977, 616-620.

[102] L. Iturrioz, O. Rueda, Algèbres implicatives trivalentes de Łukasiewicz libres, Discrete Math., 18, 1977, $35-44$.

[103] I. Leuştean, A unifying framework for Łukasiewicz-Moisil algebras, MV-algebras and Post algebras, submitted. 
[104] J. Łukasiewicz, On three-valued logic (Polish), Ruch Filozoficzny, 5, 1920, 160-171.

[105] J. Łukasiewicz, Philosophische Bemerkungen zur mehrwertigen Systemen des Aussagenkalküls, C.R. Séances Soc. Sci. Lettres Varsovie, Cl. III, 23, 1930, 51-77. Romanian translation in: Logică şi Filozofie, Ed. Politică, Bucureşti, 1966, 295-320.

[106] J. Łukasiewicz, Die Logik und das Grundlagenproblem, Les entretiens de Zurich sur les fondements et la méthode des sciences mathématiques, 1941, 88-100.

[107] J. Łukasiewicz, A. Tarski, Untersuchungen über den Aussagenkalkül, C.R. Séances Soc. Sci. Lettres Varsovie, Cl. III, 23, 1930, 30-50.

[108] M.G. Malinowski, n-valued Łukasiewicz algebras and their connection to Post algebras of order (Polish), Zeszyty Naukowe u.t., Filozofia, 1972.

[109] P. Mangani, On certain algebras related to many-valued logics (Italian), Boll. Un. Mat. Ital. (4) 8, 68-78, 1973.

[110] W. Marek, T. Traczyk, Generalized Łukasiewicz algebras, Bull. Acad. Polonaise Sci. Sér. Math. Astronom. Phys., 17, 1969, 789-792.

[111] Gr. C. Moisil, Recherches sur l'algèbre de la logique, Ann. Sci. Univ. Jassy, 22, 1935, 1-117.

[112] Gr. C. Moisil, Sur le mode problématique, C.R. Séances Acad. Sci. Roumanie, 2, No. 2, 1938, $101-103$.

[113] Gr. C. Moisil, Recherches sur les logiques non-chrysippiennes, Ann. Sci. Univ. Jassy, 26, 1940, 431-466.

[114] Gr.C. Moisil, Notes sur les logiques non-chrysippiennes, Ann. Sci, Univ. Jassy, 27, 1941, 86-98.

[115] Gr. C. Moisil, Sur les anneaux de caractéristique 2 ou 3 et leurs applications, Bul. Politechn. Bucharest, 12, 1941, 66-90.

[116] Gr. C. Moisil, Contributions à l'étude des logiques non-chrysippiennes. I. Un nouveau système d'axiomes pour les algèbres Łukasiewicziennes tétravalentes, C.R. Acad. Sci. Roumanie, 5, 1941, 289-293.

[117] Gr. C. Moisil, Contributions à l'étude des logiques non-chrysippiennes. II. Anneaux engendrés par les algèbres Łukasiewicziennes centrées, C.R. Acad. Sci. Roumanie, 6, 1942, 9-14.

[118] Gr. C. Moisil, Contributions à l'étude des logiques non-chrysippiennes. III. Anneaux engendrés par les algèbres Łukasiewicziennes tétravalentes axées, C.R. Acad. Sci. Roumanie, 7, 1942, 14-18.

[119] Gr. C. Moisil, Logique modale, Disquis. Math. Phys., 2, 1942, 3-98.

[120] Gr. C. Moisil, The algebra of networks with rectifiers (Romanian), Rev. Univ. C.I. Parhon şi a Politehnicii Bucureşti, 4-5, 1954, 9-41.

[121] Gr. C. Moisil, Utilization of three-valued logics in the theory of switching circuits. II. The characteristic equation of a relay. III. Actual-contact circuits. IV. Realization of the working functions in actual operation (Romanian). Comun. Acad. R.P. Române, 6, 1956, 231-239, 385-386, 971-973.

[122] Gr. C. Moisil, Applications of three-valued logics to the study of actual operation of relay-contact circuits (Romanian), Bul. Mat. Soc. St. Fiz. R.P. Române, 1(49), 1957, 147-191.

[123] Gr. C. Moisil, Utilization of three-valued logics to the theory of switching circuits. V. P-I circuits (Romanian), Comunic. Acad. R.P. Române, 8, 1958, 1127-1128.

[124] Gr. C. Moisil, Utilization of three-valued logics to the theory of switching circuits. VI. Polarized relays with unstable neutral. VII. Operation of ordinary relays under low self-maintaining current. VIII. -twoterminals with contacts and resistances. IX. -two-terminals with contacts, valves and resistances. X. Physical interpretation of the characteristic function of a multiterminal (Romanian), Comunic. Acad. R.P. Române, 9, 1959, 411-413, 531-532, 533-535, 665-666, 667-669. 
[125] Gr. C. Moisil, Sur l'application de la logique à trois valeurs à l'étude des circuits électriques à contacts, redresseurs et résistances, Rev. Math. Pures Appl. , 4, 1959, 173-183.

[126] Gr. C. Moisil, The algebraic Theory of Switching Circuits (Romanian), Ed. Tehnică Bucureşti. English translation 1969, Pergamon Press, Oxford, and Editura Tehnică Bucureşti.

[127] Gr.C. Moisil, Sur les idéaux des algèbres Łukasiewicziennes trivalentes, Analele Univ. C.I. Parhon, Seria Acta Logica, 3, 1960, 83-95.

[128] Gr.C. Moisil, On predicate calculus in three-valued logics (Russian), An. Univ. C.I. Parhon, Acta Logica, 4, 1961, 103-112.

[129] Gr.C. Moisil, Sur la logique à trois valeurs de Łukasiewicz, An. Univ. C.I. Parhon, Acta Logica, 5, 1962, 103-117.

[130] Gr.C. Moisil, Les logiques non-chrysippiennes et leurs applications, Acta Philos. Fennica, 16, 1963, 137152.

[131] Gr.C. Moisil, Le algèbre di Łukasiewicz, An. Univ. C.I. Parhon, Acta Logica 6, 1963, 97-135.

[132] Gr.C. Moisil, Applicazioni dell'algebra alle calcolatrici moderne, Atti Reunione del Groupement des Math. D’Expression Latine, 26.IX-3.X. 1961, Ed. Cremonese, Roma, 1963.

[133] Gr.C. Moisil, The interest of the actual operation of switching circuits for the logician, An. Univ. Bucureşti, Acta Logica, 7-8, 1964, 131-139.

[134] Gr.C. Moisil, Sur les logiques de Łukasiewicz à un nombre fini de valeurs, Rev. Roum. Math. Pures Appl., 9, 1964, 905-920.

[135] Gr.C. Moisil, Încercări vechi şi noi de logică neclasică (Old and New Essays on Non-Classical Logics), Edit. Ştiinţifică, Bucharest, 1965.

[136] Gr.C. Moisil, Théorie structurelle des automats finis, Gauthiers-Villars, Paris, 1967.

[137] Gr.C. Moisil, Łukasiewiczian algebras, Computing Center, University of Bucharest (preprint), 311-324, 1968.

[138] Gr.C. Moisil, Essais sur les logiques non-chrysippiennnes, Ed. Academiei R.S.R., Bucharest, 1972.

[139] Gr.C. Moisil, Ensembles flous et logiques à plusieurs valeurs, Centr. Rech. Math., Université de Montréal, mai, 1973 (preprint).

[140] Gr.C. Moisil, Lecţii despre logica raţionamentului nuanţat (Lectures on the Logic of Fuzzy Reasoning), Ed. Ştiinţifică şi Enciclopedică, Bucharest, 1975.

[141] Gr.C. Moisil, Sur l'emploi des mathématiques dans les sciences de l'homme, Accad. Naz. Lincei, Contributi del Centro Linceo Interdisciplinare di Sci. Mat. E loro Appl., No. 17, 1976.

[142] A. Monteiro, Matrices de Morgan caracteristiques pour le calcul propositional classique, Ann. Acad. Brasil, 52, 1960, 1-7.

[143] A. Monteiro, Sur la définition des algèbres de Łukasiewicz trivalentes, Notas de Logica matematica, 21, 1964.

[144] A. Monteiro, Construction des algèbres de Łukasiewicz trivalentes dans les algèbres de Boole monadiques, Notas de Logica Matematica, 11, 1964.

[145] A. Monteiro, R. Cignoli, Construccion geometrica de las algebras de Łukasiewicz trivalentes libres, Rev. Union Mat. Argentina, 22, 1965, 152-153.

[146] L. Monteiro, Axiomes indépendentes pour les algèbres de Łukasiewicz trivalentes, Notas de Logica Matematica, 32, 1974. 
[147] L. Monteiro, Algebras de Łukasiewicz trivalentes monadicas, Notas de Logica Matematica, 32, 1974.

[148] L. Monteiro, Sur la construction L des algèbres de Łukasiewicz trivalentes, Rev. Roum. Math. Pures Appl., Tome XXIII, No. 1, 1978, 77-83.

[149] L. Monteiro, L.G. Coppola, Sur une construction des algèbres de Łukasiewicz trivalentes, Notas de Logica Matematica, 17, 1964.

[150] D. Mundici, MV-algebras are categorically equivalent to bounded commutative BCK-algebras, Math. Japonica, 31, No. 6, 1986, 889-894.

[151] D. Mundici, Interpretation of $A F C^{\star}$-algebras in Łukasiewicz sentential calculus, J. Funct. Anal., 65, 1986, 15-63.

[152] D. Mundici, The $C^{*}$-algebras of three-valued logic, Logic Colloquium' 88 , Ferro, Bonotto, Valentini and Zanardo (Editors), Elsevier Science Publishers B.V. (North-Holland), 1989, 61-77.

[153] Gh. Nadiu, On a method for the construction of three-valued Łukasiewicz algebras (Romanian), Studii Cerc. Mat., 19, 1967, 1063-1070.

[154] A. Petcu, The definition of the trivalent Łukasiewicz algebras by three equations, Rev. Roumaine Math. Pures Appl., 13, 1968, 247-250.

[155] I. Petrescu (I. Voiculescu), Injective objects in the category of De Morgan algebras, Rev. Roumaine Math. Pures Appl., 16, 1971, 921-926.

[156] D. Ponasse, Algèbres floues et algèbres de Łukasiewicz, Rev. Roumaine Math. Pures Appl., XXIII, 1, 1978, 103-111.

[157] E. Post, Introduction to a general theory of elementary propositions, Amer. J. Math., 43, 1921, 163-185.

[158] E. Radu, L'oeuvre de Gr. C. Moisil en logique mathématique, I., II., Rev. Roumaine Math. Pures Appl., 23, 1978, 463-477, 605-610.

[159] J. Rodriguez, A. Torrens, Wajsberg Algebras and Post Algebras, Studia logica, 53, 1994, 1-19.

[160] P. Rosenbloom, Post algebras. I. Postulates and general theory, Amer. J. Math., 64, 1942, 167-183.

[161] S. Rudeanu, On Łukasiewicz-Moisil algebras of fuzzy sets, Studia Logica, 52, 1993, 95-111.

[162] A. Sade, Algèbres de Łukasiewicz dans la logique trivalente, Univ. Beograd, Publ. Elektrotehn. Fak. Ser. Mat. Fiz., No. 247-273, 1969, 123-130.

[163] C. Sanza, Notes on $n \times m$-valued Łukasiewicz algebras with negation, L. J. of the IGPL, 6, 12, 2004, 499-507 (<http://jigpal.oupjournals.org/current.dtl $>)$

[164] C. Sanza, Monadic $n \times m$-valued Łukasiewicz algebras with negation, Manuscript.

[165] C. Sicoe, Strictly chrysippian elements in many-valued Łukasiewicz algebra (Romanian), An. Univ. of Bucharest, 15, 1966, 123-126.

[166] C. Sicoe, Sur les ideaux des algèbres Łukasiewicziennes polivalentes, Rev. Roum. Math. Pures Appl., 12, 1967, 391-401.

[167] C. Sicoe, Note asupra algebrelor Łukasiewicziene polivalente, Stud. şi Cerc. Mat., 19, 1967, 1203-1207.

[168] C. Sicoe, On many-valued Łukasiewicz algebras, Proc. Japan Acad., 43, 1967, 725-728.

[169] C. Sicoe, A characterization of Łukasiewicz algebras. I. II. Proc. Japan Acad., 43, 1967, 729-732, 733-736.

[170] C. Sicoe, Sur la définition des algèbres Łukasiewicziennes polyvalentes, Rev. Roumaine Math. Pures Appl., 13, 1968, 1027-1030. 
[171] W. Suchon, On the non-equivalence of two definitions of the algebra of Łukasiewicz, P Polish Acad. Sci. Inst. Philos. Sociol. Bull. Sect. Logic, 1, No. 1, 1972, 35-37.

[172] W. Suchon, Inéquivalence de certaines définitions des algèbres infinites de Łukasiewicz, Rep. Math. Logic, 1, 1973, 21-26.

[173] W. Suchon, On defining Moisil's functors in $n$-valued Łukasiewicz propositional logic, Polish Acad. Sci. Inst. Philos. Sociol. Bull. Sect. Logic, 2, 1973, 195-196.

[174] W. Suchoń, Définition des foncteurs modaux de Moisil dans le calcul $n$-valent des propositions de Łukasiewicz avec implication et négation, Reports on Mathematical Logic 2, 43-48, 1974.

[175] W. Suchon, Matrix Łukasiewicz algebras, Rep. Math. Logic, 4, 1975, 91-104.

[176] W. Suchon, Définition des foncteurs modaux de Moisil dans le calcul $n$-valent des propositions de Łukasiewicz avec implication et négation, Reports on Mathematical Logic, 2, 1974, 43-48.

[177] J. Varlet, Algèbres de Łukasiewicz trivalentes, Bull. Soc. Roy. Sci. Liège, 36, 1968, 399-408.

[178] J. Varlet, Considérations sur les algèbres de Łukasiewicz trivalentes, Bull. Soc. Roy. Sci. Liège,38, 1969, 462-469.

[179] M. Wajsberg, Axiomatization of three-valued propositional calculus (Polish), C.R. Séances Soc. Sci. Lettres Varsovie, Cl. III, 24, 1931, 126-145.

[180] R. Wójcicki, A theorem on the finiteness of the degree of maximality of the $n$-valued Łukasiewicz logic, Polish Acad. Sci. Inst. Philos. Sociol. Bull. Sect. Logic, 4, 1975, 19-25.

[181] R. Wójcicki, G. Malinowski (editors), Selected Papers on Łukasiewicz sentencial calculi, Ossolineaum, Wroclaw and Warsaw, 1977.

[182] L.A. Zadeh, Fuzzy Sets, Inform. And Control, 8, 1965, 338-353.

George Georgescu University of Bucharest Address: Str. Academiei No. 14, Romania E-mail: georgescu@funinf.cs.unibuc.ro

Afrodita Iorgulescu Academy of Economic Studies Address: Piaţa Romană Nr. 6 - R 70167, Oficiul Poştal 22, Bucharest, Romania E-mail: afrodita@ase.ro

Sergiu Rudeanu University of Bucharest Address: Str. Academiei No. 14, Romania E-mail: rud@funinf.cs.unibuc.ro 